\title{
Influence of neural stem cell transplantation on angiogenesis in rats with spinal cord injury
}

\author{
Z. Li, G.-H. Guo, G.-S. Wang, C.-X. Guan and L. Yue \\ Department of Rehabilitation, \\ The Fifth Affiliated Hospital of Zhengzhou University, Zhengzhou, China \\ Corresponding author: G.-H. Guo \\ E-mail: ganghuaguo@163.com
}

Genet. Mol. Res. 13 (3): 6083-6092 (2014)

Received July 25, 2013

Accepted January 10, 2014

Published August 7, 2014

DOI http://dx.doi.org/10.4238/2014.August.7.23

\begin{abstract}
We examined the influence of neural stem cell transplantation on angiogenesis in rats with spinal cord injury. Sixty rats with spinal cord injury were divided into an experimental group and a control group and given neural stem cells or an equivalent amount of phosphate-buffered saline by intravenous transplantation, respectively. Basso, Beattie, and Bresnahan (BBB) motor function assessment was performed in rats at different times after transplantation, and von Willebrand factor (vWF) immunofluorescence and Western blot analysis of vascular endothelial growth factor (VEGF) protein were also performed. The BBB scores of rats in the 2 groups were both zero before transplantation. The BBB score gradually increased over time. The BBB score of the experimental group showed no significant difference compared with that of the control group $(\mathrm{P}>0.05) 7$ days after transplantation. The BBB score of the experimental group was significantly improved compared with that of the control group 14 days after transplantation $(\mathrm{P}<0.05)$. vWF-positive cells and VEGF protein expression in the experimental group were significantly increased compared with those in the control group 7 and 14 days after transplantation, respectively $(\mathrm{P}<0.05)$. Neural stem cell transplantation
\end{abstract}


may promote angiogenesis by inducing VEGF expression as well as improve functional recovery of limb movements.

Key words: Neural stem cells; Spinal cord injury; Angiogenesis

\section{INTRODUCTION}

Spinal cord injury causes full or partial loss of movement or sensation below the injury site(s) (Garbossa et al., 2012). Damaged nerve tissues have a very limited ability to self-repair, requiring necessary external intervention to restore lost function due to spinal cord injury (Oudega, 2012). Recent studies have indicated that angiogenesis plays a vital role in spinal cord injury repair (Reginato et al., 2011). The lack of local vascular tissue at the injury site hinders the ability of the body to self-heal and limits the use of treatment measures. Reducing blood loss, promoting new blood vessel formation, and restoring blood supply to the lesions may contribute to the recovery of spinal cord injury (Fassbender et al., 2011). One of the primary mechanisms of vascular formation and proliferation involves increased expression of vascular endothelial growth factor (VEGF). VEGF is a highly specific multifunctional cytokine affecting the vascular endothelium; the main functions of VEGF include promoting division and migration of blood vessel endothelial cells, inhibiting endothelial cell apoptosis, and promoting angiogenesis (Hicklin and Ellis, 2005). Neural stem cells (NSC) are promising candidate cells for neural transplantation therapy and have been shown to promote functional recovery after spinal cord injury through cell and tissue replacement (Sandner et al., 2012). Recent studies have shown that NSCs can not only replace damaged nerve tissue, but also promote angiogenesis in the ischemic area of cerebral infarction (Zhang et al., 2009). These cells can also promote the expression of various growth factors through paracrine signaling pathways (Ii et al., 2009). To examine the role of the NSC transplantation mechanism, we transplanted NSCs into rats with spinal cord injury in vivo. VEGF expression at the damaged zone, angiogenesis, and functional recovery were determined in rats after cell transplantation.

\section{MATERIAL AND METHODS}

\section{Experimental animals}

Sixty adult male Sprague-Dawley (SD) rats weighing $250 \pm 25 \mathrm{~g}$ and 10 newborn SD rats within $24 \mathrm{~h}$ were provided by the Experimental Animal Center of Zhengzhou University. This study was carried out in strict accordance with the recommendations in the Guide for the Care and Use of Laboratory Animals of the National Institutes of Health. The animal use protocol was reviewed and approved by the Institutional Animal Care and Use Committee (IACUC) of the Fifth Affiliated Hospital of Zhengzhou University.

\section{Culture and identification of NSCs}

Neonatal SD rats within $24 \mathrm{~h}$ were killed under sterile conditions after disinfection with $75 \%$ ethanol. The cortex area was removed, surface meninges and blood vessels were 
peeled off, and the samples were washed with phosphate-buffered saline (PBS). Samples were digested with $0.25 \%$ trypsin at $37^{\circ} \mathrm{C}$ for $10 \mathrm{~min}$, after which Dulbecco's modified Eagle's medium (DMEM)/F12 neutral medium (HyClone American Company) containing $10 \%$ fetal bovine serum (Gibco, USA) was added to terminate digestion. The sample mixture was repeatedly pipetted with a dropper into a single-cell suspension and filtered through a 200-mesh sieve. Next, 2\% B27 additives (Invitrogen; Carlsbad, CA, USA), $20 \mathrm{ng} / \mathrm{mL}$ basic fibroblast growth factor (PeproTech, Princeton, NJ, USA), and $20 \mathrm{ng} / \mathrm{mL}$ DMEM/F12 (PeproTech) containing epidermal growth factor were added to the filtered cell suspension solution, which was then centrifuged at $300 \mathrm{~g}$ for $5 \mathrm{~min}$. The supernatant was discarded and the appropriate amount of culture medium was added; the mixture was repeatedly pipetted into a single cell suspension and cell density was adjusted to $5 \times 10^{5} / \mathrm{mL}$, followed by inoculation into $25-\mathrm{mL}$ flasks. Cells were incubated at $37^{\circ} \mathrm{C}$ and $5 \% \mathrm{CO}_{2}$. The medium was changed once every 3-4 days and passaged once every 5-7 days based on cell growth.

The suspension of cultured third-generation NSCs showing good growth was seeded on polylysine-treated 12-well cell culture plates with coverslips and cultured for $4 \mathrm{~h}$. Nestin immunofluorescence of NSCs was performed when cell ball adherence was observed. The specific steps were as follows: the coverslips containing the cell samples from the culture plates were removed, washed with $\mathrm{PBS}$, fixed with $4 \%$ paraformaldehyde at $4{ }^{\circ} \mathrm{C}$ for $30 \mathrm{~min}$, and washed with PBS 3 times for 5 min each time. Membranes were ruptured with $0.4 \%$ TritonX-100 (Beijing Soledad Technology Co., Ltd., Beijing, China) for 30 min, washed with PBS 3 times for 5 min each time, goat serum-mounted for $30 \mathrm{~min}$ at room temperature, incubated with rabbit anti-rat Nestin polyclonal antibody (1:200; Shanghai Biological Technology Company, Shanghai, China), incubated overnight in a $4^{\circ} \mathrm{C}$ wet box, washed with PBS 3 times for $5 \mathrm{~min}$ each time, incubated with fluorescein isothiocyanate-labeled goat anti-rabbit secondary antibody (1:150; Beijing Zhongshan Golden Bridge Biotechnology, Beijing, China), and incubated for $2 \mathrm{~h}$ at room temperature and washed with PBS 3 times for $5 \mathrm{~min}$ each. Slices were mounted in neutral glycerol, observed, and photographed using a fluorescence microscope (BX41 Olympus, Tokyo, Japan).

\section{Spinal cord injury model preparation and NSC transplantation}

Rats were anesthetized using $0.38 \mathrm{~mL} / 100 \mathrm{~g}(10 \%)$ chloral hydrate by intraperitoneal injection. Rats were fixed in the prone position, disinfected, and draped, and other routine pre-operative preparations were performed. A $2-\mathrm{cm}$ longitudinal incision was made along the T7-T11 vertebral midline, the skin and paraspinal muscles were cut layer by layer, and the T8T10 spinous process and vertebral plate were removed while maintaining the integrity of the endocranium. The spinal cord was injured using a modified Allen method. Partial spinal cord edema appeared after hitting. Rats with spasmodic reflex, wagging tail, and flaccid paralysis after retraction of the flutter appeared in the 2 limbs were considered to be successfully prepared. After operation, hemostasis and suturing of the muscle and incisions layer by layer of the rats are needed. Penicillin injections were given after 3 consecutive days to prevent infections. The bladder was massaged twice a day to promote urination until spontaneous voiding was restored. After $24 \mathrm{~h}, 500 \mu \mathrm{L}$ neural stem cell suspension (cell concentration was $1 \times 10^{7 /}$ $\mathrm{mL}$ ) was transplanted into experimental group rats through tail venous injection; the same volume of PBS was given to the rats in the control group. 


\section{Western blotting}

Seven and 14 days after transplantation, 15 rats from each group were randomly selected, $10 \%$ chloral hydrate anesthetized, and supine-fixed to expose the heart. Rats were intubated through the left ventricular catheter, the right atrial appendage was cut and quickly rinsed with $200 \mathrm{~mL}$ normal saline, and perfused with $4 \%$ paraformaldehyde. Next, 1-cm tissue blocks (taking the injury plane as the center) were placed in 4\% paraformaldehyde and fixed, and part of the tissue was dehydrated using a sucrose gradient for immunofluorescence after $24 \mathrm{~h}$. The rest of the sample was frozen in liquid nitrogen for Western blotting.

Ice-cold radioimmunoprecipitiation assay lysis buffer was added to $20 \mathrm{mg}$ frozen tissue and the homogenate was ground on ice, transferred to a precooled microcentrifuge tube, and placed on ice for $1 \mathrm{~h}$ until full dissociation had occurred. The lysate was centrifuged at $14,000 \mathrm{rpm}$ at $4^{\circ} \mathrm{C}$ for $30 \mathrm{~min}$. The supernatant was collected and protein was quantified using the bicinchoninic acid method. In addition, $50 \mu \mathrm{g}$ protein per well of sample volume was run on $10 \%$ sodium dodecyl sulfate-polyacrylamide gel electrophoresis (SDS-PAGE) for electrophoretic separation, and the proteins were transferred to a polyvinylidene fluoride membrane, and incubated in 5\% skim milk at room temperature for $1 \mathrm{~h}$. Primary rabbit anti-rat anti-VEGF antibody (1:200; Shanghai Sangon Biotech Corporation, Shanghai, China) was added and the membrane was incubated overnight at $4{ }^{\circ} \mathrm{C}$. The conjugated secondary antibody was added for 1 $\mathrm{h}$ after washing the membrane and transferred to X-ray film using the enhanced chemiluminescence method. The membrane was analyzed using the Gel-Pro 4.0 version gel optical analysis software, the absorbance of the target protein and the standard control were measured, and the relative gray value was calculated.

\section{Immunofluorescence detection}

Seven and 14 days after injury, von Willebrand factor (vWF) immunofluorescent staining was performed. vWF-positive cells located in vascular tissue-dense areas of the damaged area were observed using fluorescence microscopy, counted, and averaged. The immunofluorescence steps were as follows: sagittal slices were made at $-25^{\circ} \mathrm{C}$ using a freezing microtome and slice thickness was adjusted to $10 \mu \mathrm{m}$. Slices were dried at room temperature, PBS-washed for $5 \mathrm{~min} 3$ times, incubated with $0.4 \%$ Triton X-100 (PBS preparation) at room temperature for 30 min, PBS-washed for 5 min 3 times, incubated with $10 \%$ bovine serum albumin antigen at room temperature for $30 \mathrm{~min}$, incubated with mouse anti-rat vWF multi-clonal antibody (1:500; Santa Cruz Biotechnology, Inc., Santa Cruz, CA, USA), incubated in a wet box at $4^{\circ} \mathrm{C}$ overnight, PBS-washed for 5 min 3 times, incubated with TRITC-labeled goat anti-mouse secondary antibody (1:150; Beijing Zhongshan Golden Bridge Biotechnology Company), incubated at $37^{\circ} \mathrm{C}$ without light for $2 \mathrm{~h}$, PBS-washed for $5 \mathrm{~min} 3$ times, and placed in glycerol. Images were acquired using fluorescence microscopy.

\section{Comparison of rat Basso, Beattie, and Bresnahan (BBB) motor function scoring in groups}

Motor function of the rat hind limbs in each group was scored using the BBB scoring 
method (Falconer et al., 1996) before transplantation and at 7 and 14 days after transplantation. Rats without fatigue were scored at the same time every day. Rats were placed in an open space of $125 \times 125 \mathrm{~cm}$, allowed to move freely, and were lured using articles. Parameters such as hind limb movement, body control, and coordination were observed after spinal cord injury for a total of $4 \mathrm{~min}$.

\section{Statistical analysis}

Data are reported as means \pm SD and statistical analysis was performed using the SPSS17.0 statistical software (SPSS, Inc., Chicago, IL, USA). P $<0.05$ was considered to be statistically significant, and BBB score, number of vWF-positive cells, and expression levels of VEGF protein between groups or among different times were compared using the Student $t$-test of paired samples.

\section{RESULTS}

\section{Cell culture and identification}

The single-cell suspension with cytoplast optically transparent was observed under a microscope after 3 days of culture. The single-cell suspension gradually decreased 5-7 days later, and the groups were gathered to form stem cell spheres of various sizes. Cells in the middle of the spheres were of regular morphology with clear boundaries and strong refractivity (Figure 1). Strong positive green fluorescence was observed in the cytoplasm after Nestin immunofluorescence staining, while the nuclei remained unstained (Figure 2).

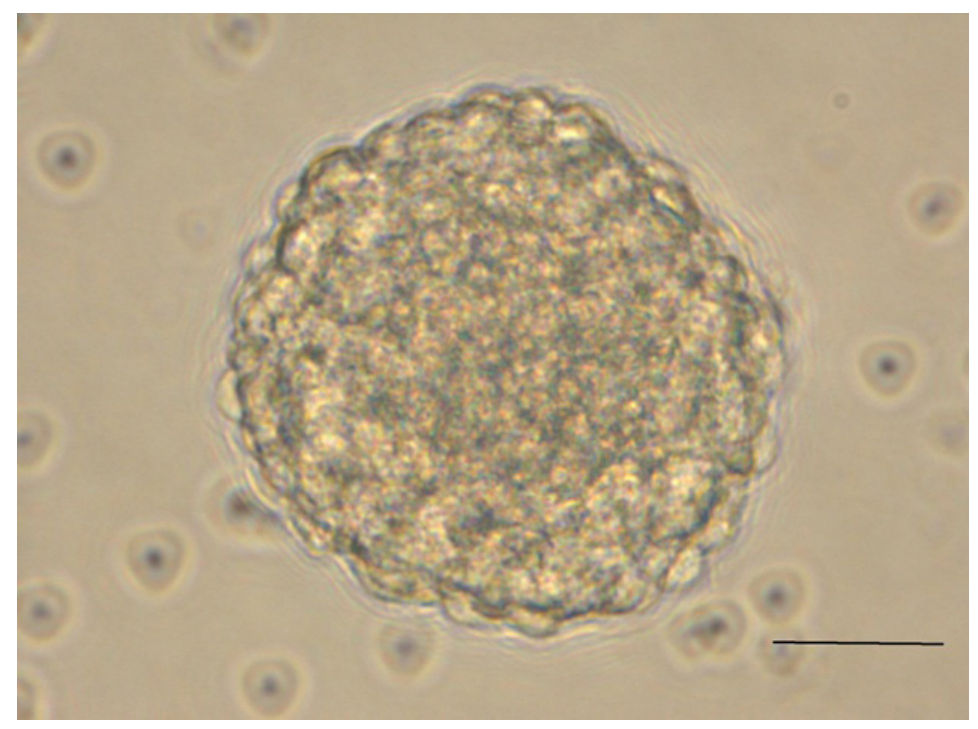

Figure 1. Neural stem cell spheres cultured for 7 days. Scale bar $=100 \mu \mathrm{m}$. 


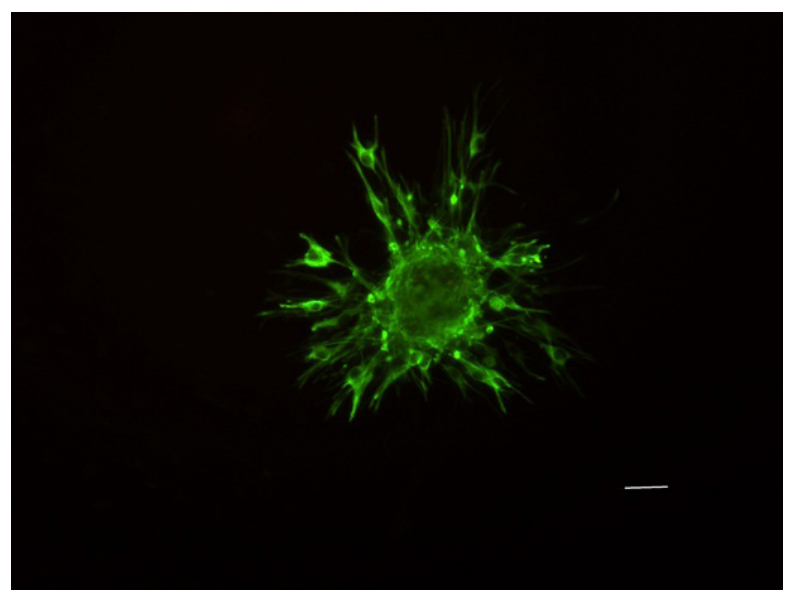

Figure 2. Nestin fluorescence of neural stem cell spheres. Scale bar $=100 \mu \mathrm{m}$.

\section{Comparison of BBB scores at different times in spinal cord injury rats}

BBB scoring for the spinal cord injury rats was performed for rats in the 2 groups at the same time 1 day after spinal cord injury (before transplantation) and 7 and 14 days after transplantation. The BBB score of rats in both groups 1 day after spinal cord injury (before transplantation) was 0 , while the BBB score of the rat hind legs in both groups 7 days after transplantation gradually improved; all hind leg function was improved, but there were no significant differences between the NSC group and the PBS group $(\mathrm{P}>0.05)$. The BBB score gradually increased over time and was significantly improved in the NSC group compared with the PBS group 14 days after transplantation $(\mathrm{P}<0.05$; Table 1$)$.

Table 1. BBB score of hind limb function at different times after spinal cord injury in the two groups.

\begin{tabular}{lcccc}
\hline Group & $\mathrm{N}$ & 1 day & 7 days & 14 days \\
\hline PBS group & 30 & 0 & $2.36 \pm 0.92$ & $5.19 \pm 1.16$ \\
NSC group & 30 & 0 & $2.76 \pm 0.85$ & $6.65 \pm 1.08$ \\
F value & & & 0.656 & 0.122 \\
P value & & & 0.404 & 0.000 \\
\hline
\end{tabular}

Data are reported as means \pm SD. PBS $=$ phosphate-buffered saline; NSC $=$ neural stem cell. BBB score of the NSC group was not significant different compared with that of the control group 7 days after transplantation $(\mathrm{P}>$ 0.05). BBB score of the NSC group was significantly different compared with that of the control group 14 days after transplantation $(\mathrm{P}<0.05)$.

\section{Comparison of VEGF protein expression in the damaged area at different times}

The relative expression of VEGF protein in the spinal cord in the PBS group was $0.53 \pm 0.013$ and $0.414 \pm 0.016$ at 7 and 14 days after transplantation, respectively (Figure 3 and Table 2). The relative expression of VEGF protein in the spinal cord in the PBS group gradually decreased over time. The relative expression of VEGF protein in the spinal cord in the NSC group was $0.648 \pm 0.017$ and $0.688 \pm 0.023$ at the corresponding times, which was higher than that in the PBS group; this difference was statistically significant $(\mathrm{P}<0.05)$. The relative expression of VEGF protein for the spinal cord in the NSC group at 14 days was 
higher than that at 7 days, and the difference was statistically significant $(\mathrm{P}<0.05$; Table 2$)$.

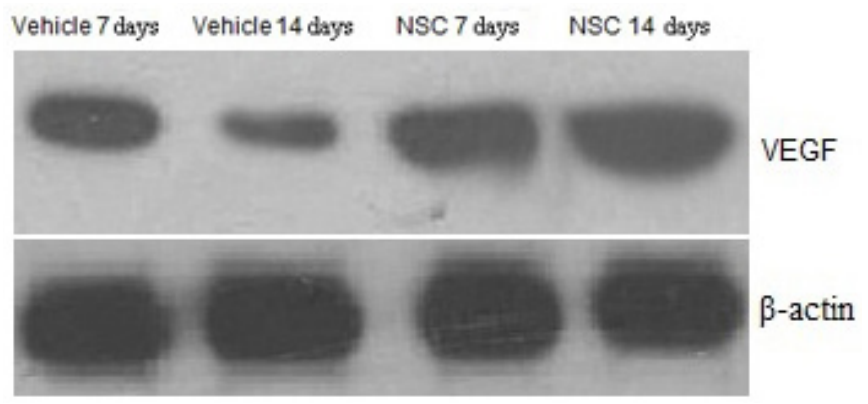

Figure 3. Western blot analysis of VEGF protein expression.

Table 2. Relative expression amount of VEGF protein (VEGF/GAPDH) in rats of the two groups at different times.

\begin{tabular}{lccc}
\hline Group & $\mathrm{N}$ & 7 days & 14 days \\
\hline PBS group & 30 & $0.536 \pm 0.013$ & $0.414 \pm 0.016$ \\
NSC group & 30 & $0.648 \pm 0.017$ & $0.688 \pm 0.023$ \\
F value & & 4.473 & 6.289 \\
P value & & 0.000 & 0.000 \\
\hline
\end{tabular}

Data are reported as means $\pm \mathrm{SD}$. PBS $=$ phosphate-buffered saline; NSC = neural stem cell. The NSC group was significantly different compared with the PBS group.

\section{Comparison of angiogenesis in the damaged zone at different times}

Spinal cord injury rats were killed at 7 and 14 days after NSC transplantation. The results of vWF immunofluorescence staining to detect lesions (Figure 4) showed that the number of positive vessels in the PBS group at 7 and 14 days were $39.66 \pm 5.38$ and $42.10 \pm 5.38$, respectively, and $54.01 \pm 7.38$ and $59.32 \pm 5.18$ for the NSC group, respectively. These values were significantly increased compared to the control group $(\mathrm{P}<0.05)$, while the number of vWF-positive vessels in the experimental group at 14 days was significantly higher than at 7 days $(\mathrm{P}<0.05$; Table 3$)$.

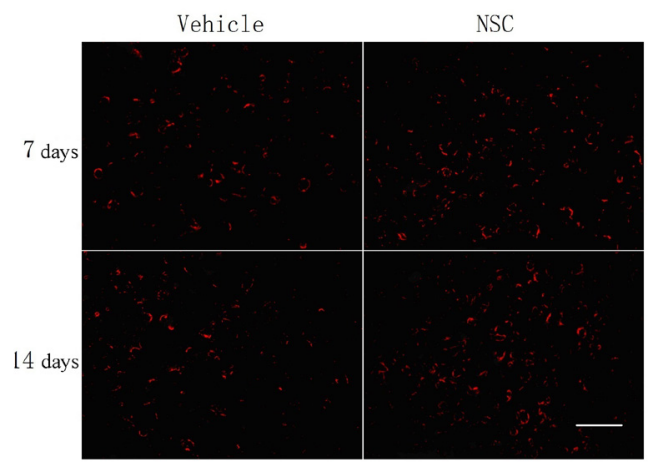

Figure 4. Number of vWF-positive blood vessels at 7 and 14 days in NSC and control groups. NSC $=$ neural stem cell. Scale bar $=100 \mu \mathrm{m}$. 
Table 3. vWF-positive blood vessels in rats of the two groups at different times.

\begin{tabular}{lccc}
\hline Group & $\mathrm{N}$ & 7 days & 14 days \\
\hline PBS group & 30 & $39.66 \pm 5.38$ & $42.10 \pm 5.38$ \\
NSC group & 30 & $54.01 \pm 7.38$ & $59.32 \pm 5.18$ \\
F value & & 1.426 & 0.232 \\
P value & & 0.000 & 0.000 \\
\hline
\end{tabular}

Data are reported as means \pm SD. PBS $=$ phosphate-buffered saline; NSC $=$ neural stem cell. The NSC group was significantly different compared with the PBS group.

\section{DISCUSSION}

In general, in a highly active metabolic state, the absence of blood vessels may limit tissue recovery or regeneration. Promoting angiogenesis can be used to treat low levels of vascular tissue caused by disease or trauma (Mitsos et al., 2012). Clinically, angiogenesis therapy is primarily used to treat cardiovascular disease (Mitsos et al., 2012; Won et al., 2013), while anti-angiogenic therapy is used to treat cancer and malignant tumors (Shojaei, 2012). Most previous studies that have examined tissue repair after spinal cord injury concentrated on nerve regeneration (Ronaghi et al., 2010), while the neuroprotective effect of microvascular regeneration remains underexplored. Recent studies have indicated that angiogenesis plays a very important role in axonal regeneration after spinal cord injury. In addition to relying on nerve fiber regeneration and synaptic reconstruction, tissue repair and functional recovery after spinal cord injury require nutritional support provided by blood vessels to nourish damaged tissues (Oudega, 2012). Promoting the formation of blood vessels near the site of injury and providing the environment necessary for cell survival may promote axonal regeneration, thereby improving treatment efficacy. Various studies have indicated that promoting nerve tissue angiogenesis after injury is a potential strategy for restoring impaired function (Kundi et al., 2013). Intervention by drugs or cells for improving angiogenesis was shown to promote functional recovery (Han et al., 2012; Oh et al., 2012). As a potent angiogenic stimulus and vascular permeability regulation factor (Ferrara et al., 2003), VEGF has been shown to promote angiogenesis after spinal cord injury, and also plays a role in neuroprotection (Widenfalk et al., 2003). Herrera et al. (2010) reported that promoting angiogenesis after spinal cord injury improved movement recovery when exogenous VEGF was added. Due to its angiogenic and neuroprotective effects, VEGF may play a supporting role in angiogenic therapy for the spinal cord after injury.

In the present study, the effects of neural stem cell transplantation on the functional recovery after spinal cord injury were evaluated based on BBB motor scoring, VEGF secretion, and angiogenesis. The results showed that after neural stem cell transplantation of rats with spinal cord injury, all BBB motor scores, VEGF secretion, and angiogenesis increased over time compared with values in the control group. Over the past two decades, in many spinal cord injury small animal models, it was demonstrated that neural stem cell transplantation could promote functional recovery after spinal cord injury by a variety of functional recovery mechanisms, including the growth of myelin and axon regeneration (Garbossa et al., 2012; Sandner et al., 2012; Li and Lepsk, 2013). Using a spinal cord injury model, Abematsu (2010) and Amemori (2013) showed that neural stem cell transplantation could improve spinal cord injury symptoms through different mechanisms and promote the functional recovery of hind limbs. The results of this study also confirmed that over time, the BBB score of rats with spinal 
cord injury, both in the control group and in the experimental group, gradually increased, but the scores increased more in the stem cell transplantation group with faster functional recovery, confirming that NSCs can migrate to the damage sites and survive, improve hindlimb function, and promote neurological recovery.

Recent studies examining NSCs primarily concentrated on promoting nerve regeneration, including promoting endogenous NSC regeneration of the damaged body, providing neuroprotection, and differentiating into neurons and glial cells, which result in cell replacement (Karimi-Abdolrezaee and Eftekharpour, 2012; Sandner et al., 2012; Li and Lepski, 2013). Recent study has also shown that in a rat model of cerebral infarction, NSC transplantation not only provided a more favorable microenvironment for endogenous NSC proliferation and cell death inhibition through paracrine pathways, but also promoted angiogenesis (Zhang et al., 2011). This may be related to the release of various nutritional factors from NSCs, such as epidermal growth factor, fibroblast growth factor, and VEGF (Zhang et al., 2009). As a highly specific multifunctional cytokine that affects the vascular endothelium, VEGF functions in endothelial cell apoptosis inhibition, promotion of angiogenesis, and neuroprotection to promote the repair of spinal cord injury (Liu et al., 2010).

We here demonstrated that after NSC transplantation, VEGF protein and vWF-positive cells in the damaged zone were markedly increased, suggesting that angiogenesis in the damaged zone is induced by promoting VEGF expression at the spinal cord injury sites after NSC transplantation. This improved ischemia, thereby promoting body repair. Previous study (Oudega, 2012) showed that the ideal time of angiogenic therapy intervention based on VEGF and NSC was presumed to be 2 days to 2 weeks after the acute spinal cord injury. The results showed that vWF-positive cells at injury sites increased 7 days after NSC transplantation and lasted for at least 14 days.

In conclusion, we found that NSC transplantation promoted angiogenesis and improved the blood supply to the injury sites after spinal cord injury, which may be associated with post-transplant induction of VEGF secretion. Angiogenesis of the damaged zone was promoted after NSC transplantation, thereby improving ischemia in the damaged area. The promotion of nerve regeneration and angiogenesis induced by NSCs may be a treatment method for spinal cord injury. The role of VEGF in angiogenesis after spinal cord injury may result from neuroprotection mechanisms, and these processes should be further examined.

\section{ACKNOWLEDGMENTS}

Research supported by the Key Scientific and Technological Research Projects of Henan Province (\#082300450200).

\section{REFERENCES}

Abematsu M, Tsujimura K, Yamano M, Saito M, et al. (2010). Neurons derived from transplanted neural stem cells restore disrupted neuronal circuitry in a mouse model of spinal cord injury. J. Clin. Invest. 120: 3255-3266.

Amemori T, Romanyuk N, Jendelova P, Herynek V, et al. (2013). Human conditionally immortalized neural stem cells improve locomotor function after spinal cord injury in the rat. Stem Cell Res. Ther. 4: 68.

Falconer JC, Narayana PA, Bhattacharjee M and Liu SJ (1996). Characterization of an experimental spinal cord injury model using waveform and morphometric analysis. Spine 21: 104-112.

Fassbender JM, Whittemore SR and Hagg T (2011). Targeting microvasculature for neuroprotection after SCI. Neurotherapeutics 8: 240-251. 
Ferrara N, Gerber HP and LeCouter J (2003). The biology of VEGF and its receptors. Nat. Med. 9: 669-676.

Garbossa D, Boido M, Fontanella M, Fronda C, et al. (2012). Recent therapeutic strategies for spinal cord injury treatment: possible role of stem cells. Neurosurg. Rev. 35: 293-311.

Han X, Yang N, Cui Y, Xu Y, et al. (2012). Simvastatin mobilizes bone marrow stromal cells migrating to injured areas and promotes functional recovery after spinal cord injury in the rat. Neurosci. Lett. 521: 136-141.

Herrera JJ, Sundberg LM, Zentilin L, Giacca M, et al. (2010). Sustained expression of vascular endothelial growth factor and angiopoietin-1 improves blood-spinal cord barrier integrity and functional recovery after spinal cord injury. $J$. Neurotrauma 27: 2067-2076.

Hicklin DJ and Ellis LM (2005). Role of the vascular endothelial growth factor pathway in tumor growth and angiogenesis. J. Clin. Oncol. 23: 1011-1027.

Ii M, Nishimura H, Sekiguchi H, Kamei N, et al. (2009). Concurrent vasculogenesis and neurogenesis from adult neural stem cells. Circ. Res. 105: 860-868.

Karimi-Abdolrezaee S and Eftekharpour E (2012). Stem cells and spinal cord injury repair. Adv. Exp. Med. Biol. 760: 53-73.

Kundi S, Bicknell R and Ahmed Z (2013). The role of angiogenic and wound-healing factors after spinal cord injury in mammals. Neurosci. Res. 76: 1-9.

Li J and Lepski G (2013). Cell transplantation for spinal cord injury: a systematic review. Biomed. Res. Int. 2013: 786475.

Liu Y, Figley S, Spratt SK, Lee G, et al. (2010). An engineered transcription factor which activates VEGF-A enhances recovery after spinal cord injury. Neurobiol. Dis. 37: 384-393.

Mitsos S, Katsanos K, Koletsis E, Kagadis GC, et al. (2012). Therapeutic angiogenesis for myocardial ischemia revisited: basic biological concepts and focus on latest clinical trials. Angiogenesis 15: 1-22.

Oh JS, Park IS, Kim KN, Yoon DH, et al. (2012). Transplantation of an adipose stem cell cluster in a spinal cord injury. Neuroreport 23: 277-282.

Oudega M (2012). Molecular and cellular mechanisms underlying the role of blood vessels in spinal cord injury and repair. Cell Tissue Res. 349: 269-288.

Reginato S, Gianni-Barrera R and Banfi A (2011). Taming of the wild vessel: promoting vessel stabilization for safe therapeutic angiogenesis. Biochem. Soc. Trans. 39: 1654-1658.

Ronaghi M, Erceg S, Moreno-Manzano V and Stojkovic M (2010). Challenges of stem cell therapy for spinal cord injury: human embryonic stem cells, endogenous neural stem cells, or induced pluripotent stem cells? Stem Cells 28: 93-99.

Sandner B, Prang P, Rivera FJ, Aigner L, et al. (2012). Neural stem cells for spinal cord repair. Cell Tissue Res. 349: 349-362.

Shojaei F (2012). Anti-angiogenesis therapy in cancer: current challenges and future perspectives. Cancer Lett. 320: 130137.

Widenfalk J, Lipson A, Jubran M, Hofstetter C, et al. (2003). Vascular endothelial growth factor improves functional outcome and decreases secondary degeneration in experimental spinal cord contusion injury. Neuroscience 120: 951-960.

Won YW, McGinn AN, Lee M, Nam K, et al. (2013). Post-translational regulation of a hypoxia-responsive VEGF plasmid for the treatment of myocardial ischemia. Biomaterials 34: 6229-6238.

Zhang P, Li J, Liu Y, Chen X, et al. (2009). Transplanted human embryonic neural stem cells survive, migrate, differentiate and increase endogenous nestin expression in adult rat cortical peri-infarction zone. Neuropathology 29: 410-421.

Zhang P, Li J, Liu Y, Chen X, et al. (2011). Human embryonic neural stem cell transplantation increases subventricular zone cell proliferation and promotes peri-infarct angiogenesis after focal cerebral ischemia. Neuropathology 31: 384-391. 